\title{
ATIVIDADE OVICIDA DO ÓLEO ESSENCIAL E DO EXTRATO HIDROALCOÓLICO DE Ocimum basilicum SOBRE NEMATÓDEOS GASTRINTESTINAIS DE OVINOS
}

Recebido: $27 / 05 / 2017$

\author{
CASTRO, Leonardo Mortagua de ${ }^{1}$; \\ PINTO, Natália Berne ${ }^{2}$; \\ MOTA, Tairan Ourique ${ }^{3}$; \\ MOURA, Micaele Quintana de ${ }^{2}$; \\ DIAS DE CASTRO, Luciana Laitano ${ }^{4}$; \\ MADRID, Isabel Martins ${ }^{5}$; \\ FREITAG, Rogério Antônio ${ }^{6}$; \\ BERNE, Maria Elizabeth Aires ${ }^{7}$.
}

Aceito: 02/09/2017

${ }^{1}$ Doutorando do Programa de Pós-Graduação em Veterinária/UFPEL; ${ }^{2}$ Doutoranda do Programa de PósGraduação em Parasitologia/UFPEL; ${ }^{3}$ Acadêmico da Faculdade de Veterinária/UFPEL; ${ }^{4}$ Doutoranda do Programa de Pós-Graduação em Ciências Veterinárias/UFPR; ${ }^{5}$ Médica Veterinária, Prefeitura Municipal de Pelotas; ${ }^{6}$ Professor, Instituto de Química e Geociências/UFPEL; ${ }^{7}$ Professora, Instituto de Biologia/UFPEL.

\section{RESUMO}

$\mathrm{O}$ controle dos helmintos gastrintestinais em ovinos, realizado exclusivamente através de anti-helmínticos, tem como consequência a seleção de populações de helmintos resistentes a praticamente todos os fármacos disponíveis. Isso tem motivado à busca de alternativas para controle, entre as quais destacamos a utilização de fitoterápicos. Neste contexto, o objetivo deste estudo foi avaliar a atividade ovicida in vitro do óleo essencial e do extrato hidroalcoólico de Ocimum basilicum em nematódeos de ovinos e determinar os constituintes químicos majoritários do óleo essencial. Para os estudos in vitro foram utilizadas fezes de ovinos naturalmente infectados, testadas frente ao óleo essencial de $O$. basilicum nas concentrações de 32 a $0,25 \mathrm{mg} / \mathrm{mL}$ e ao extrato hidroalcoólico nas concentrações 40 a $1,25 \mathrm{mg} / \mathrm{mL}$. Como controle positivo foi utilizado o princípio ativo tiabendazol $(0,025 \mathrm{mg} / \mathrm{mL})$ e negativo a água destilada. A análise química do óleo essencial determinou como constituintes majoritários chavicol (34,68\%) e linalool (27,68\%). 0 óleo essencial apresentou atividade ovicida acima de $99 \%$ em todas as concentrações testadas. 0 extrato hidroalcoólico nas concentrações entre 40 e $20 \mathrm{mg} / \mathrm{mL}$ obteve $100 \%$ de ação, decrescendo a partir de $10 \mathrm{mg} / \mathrm{mL}$, atingindo $9,76 \%$ de atividade na concentração 1,25 $\mathrm{mg} / \mathrm{mL}$. Os controles, positivo e negativo, obtiveram $98,57 \%$ e $8,5 \%$, respectivamente. $O$ valor da concentração letal média $\left(\mathrm{CL}_{50}\right)$ do extrato hidroalcoólico e óleo essencial foram de $4,797 \mathrm{mg} / \mathrm{mL}$ e $0,2123 \mathrm{mg} / \mathrm{mL}$, respectivamente. O. basilicum apresentou ação antihelmíntica in vitro sobre ovos de nematódeos gastrintestinais de ovinos, com maior potencial para o óleo essencial, constituindo um promissor fitoterápico para estudos visando à busca de moléculas bioativas para serem utilizadas no controle dos nematódeos gastrintestinais.

Palavras-chave: Manjericão. Haemonchus. In vitro. Atividade anti-helmíntica. 


\section{INTRODUÇÃO}

A ovinocultura vem se fortalecendo nos últimos anos, com a valorização da carne e da lã e com isso firma-se como uma área importante de investimento dentro da pecuária. Entretanto, as nematodioses gastrintestinais têm acarretado prejuízos que muitas vezes inviabilizam a produção desses pequenos ruminantes (ECHEVARRIA et al., 1996; GITHIGIA et al., 2001).

O controle dessas parasitoses em ovinos vem sendo realizada através do uso de antihelmínticos sintéticos, o que tem acarretado o surgimento de populações de nematódeos resistentes e consequentemente um controle ineficiente (ALMEIDA et al., 2010). Aliado a isto, produtos de origem animal sem a presença de resíduos químicos constituem uma exigência cada vez maior dos consumidores. Entre os métodos de controle alternativos pesquisados, vem se destacando a validação de fitoterápicos, com resultados promissores em nematódeos gastrintestinais de ovinos (ASSIS et al., 2003; CARVALHO et al., 2012; GITHIORI et al., 2002; OLIVEIRA et al., 2009).

Ocimum basilicum (manjericão) é uma planta da família Lamiaceae cultivada em quase todo o Brasil, muito utilizada como condimento (BOWN, 1995). Estudos avaliando seu potencial de uso no controle de protozoários (ALMEIDA et al., 2007; MOTA et al., 2012), fungos (DAMBOLENA et al., 2010), insetos (GHOSH et al., 2012) e nematódeos (CARVALHO et al., 2012) vêm sendo realizados em diferentes regiões do mundo.

Considerando a iminente necessidade de buscar medidas alternativas de controle dos nematódeos gastrintestinais, e sendo os extratos vegetais promissores a esta finalidade, foi proposto o presente estudo. Este tem como objetivo verificar a ação in vitro do óleo essencial e do extrato hidroalcóolico de $O$. basilicum sobre ovos de nematódeos gastrintestinais de ovinos, determinar a concentração letal média $\left(\mathrm{CL}_{50}\right)$, bem como quantificar os principais componentes do óleo essencial. 


\section{MATERIAL E MÉTODOS}

\section{Recuperação dos ovos}

Amostras de fezes foram colhidas diretamente da ampola retal de dois cordeiros, naturalmente parasitados por nematódeos gastrintestinais. Os ovos foram quantificados (GORDON; WHITLOCK, 1939) e as larvas posteriormente identificadas (ROBERTS; O'SULLIVAN, 1950). A recuperação dos ovos se deu através da técnica de Hubert e Kerboeuf (1992) com modificações, sendo que as amostras positivas foram maceradas e acrescentadas de água a $40{ }^{\circ} \mathrm{C}$, seguindo-se a passagem das fezes em quatro tamises com os seguintes diâmetros de abertura de malhas: $1 \mathrm{~mm}, 105 \mu \mathrm{m}, 55 \mu \mathrm{m}$ e $25 \mu \mathrm{m}$. Os ovos retidos no último tamis foram recolhidos através de lavagem com água destilada. Este material foi transferido para tubos de $50 \mathrm{~mL}$ e centrifugado a $3000 \mathrm{rpm}$, em temperatura ambiente, por cinco minutos. O sobrenadante foi descartado e o sedimento suspenso com solução salina saturada e novamente centrifugado por cinco minutos. Após a centrifugação o sobrenadante foi novamente passado em tamis de $25 \mu \mathrm{m}$, seguindo-se a quantificação dos ovos.

\section{Obtenção do óleo essencial e extrato hidroalcoólico de 0 . basilicum}

As folhas secas de 0 . basilicum utilizadas no teste foram adquiridas de distribuidor comercial, com certificação de qualidade e origem. Para obtenção do óleo essencial, as folhas secas foram submetidas à extração com arraste de vapor em aparelho Clevenger, durante 4 h. O óleo obtido foi seco com sulfato de sódio anidro P. A., armazenado em frasco âmbar e mantido a $18{ }^{\circ} \mathrm{C}$ até a utilização.

Para a obtenção do extrato hidroalcoólico, inicialmente foi preparada a tintura de $O$. basilicum, para isso, foram pesados $100 \mathrm{~g}$ da planta e adicionados $1000 \mathrm{~mL}$ de solução alcoólica a $70 \%$ e posteriormente esta mistura foi acondicionada ao abrigo da luz sob agitação diária durante sete dias. A seguir realizou-se a filtragem, obtendo-se $760 \mathrm{~mL}$ da tintura, a qual foi acrescentado $240 \mathrm{~mL}$ de álcool 70\%. Posteriormente, foi utilizado um evaporador rotativo com pressão negativa de $600 \mathrm{mmHg}$, a uma temperatura de $45{ }^{\circ} \mathrm{C}$, com velocidade suficiente para homogeneizar e aquecer o conteúdo de maneira uniforme, resultando na evaporação da porção alcoólica da tintura. 
O óleo essencial de $O$. basilicum foi submetido à análise por cromatografia gasosa acoplada a espectrometria de massa, em um equipamento GC/MS Schimadzu QP2010, com injetor split/splitless com coluna capilar Rtx-5MS RESTEK (30 $\mathrm{m} \times 0,25 \mathrm{~mm} \times 0,25 \mu \mathrm{m})$, nas seguintes condições cromatográficas: gás carreador Hélio, fragmentos obtidos por impacto de elétrons na energia de $70 \mathrm{~V}$, vazão de $1,2 \mathrm{~mL} / \mathrm{min}$, split 1:10, volume injetado de amostra $1 \mu \mathrm{L}$. Temperatura programada do forno: a temperatura inicial foi de $40{ }^{\circ} \mathrm{C}$, com rampa de aquecimento em $5{ }^{\circ} \mathrm{C} / \mathrm{min}$ até $280{ }^{\circ} \mathrm{C}$, ficando estável nessa temperatura por 10 minutos, com tempo total de 58 minutos, sendo a temperatura do injetor de $58{ }^{\circ} \mathrm{C}$ e interface $200{ }^{\circ} \mathrm{C}$, os compostos foram analisados com base na biblioteca NIST08 do GC/MS. O óleo foi diluído em hexano P. A.

O efeito citotóxico do óleo essencial foi testado através do ensaio de MTT (brometo tiazoli azul de tetrazólio), conforme descrito por Mosmann (1983). Para isto foram utilizadas células de rim bovino Madin-Darby (MDBK).

\section{Avaliação da inibição da eclosão}

A avaliação do óleo essencial e do extrato hidroalcoólico foi realizada utilizando placas de cultivo de 24 poços com aproximadamente 150 ovos por orifício. O óleo essencial foi testado em oito concentrações $(32,16,8,4,2,1,0,5$ e $0,25 \mathrm{mg} / \mathrm{mL})$ e o extrato hidroalcoólico em seis concentrações $(40,20,10,5,2,5$ e $1,25 \mathrm{mg} / \mathrm{mL})$. Como controle positivo foi utilizado cloridrato de tiabendazol na concentração de $0,025 \mathrm{mg} / \mathrm{mL}$ e como controle negativo água destilada. Todas as avaliações foram realizadas em quatro repetições, seguindo-se de incubação em estufa B. O. D. a $28{ }^{\circ} \mathrm{C}$ com umidade relativa de $80 \%$ por 24 horas. A quantificação de ovos e larvas foi realizada em microscópio invertido (Zeiss, Alemanha). As médias da inibição da eclosão para cada tratamento foi determinada conforme a equação (CAMURÇA-VASCONCELOS et al., 2007):

número de larvas

\% de inibição da eclosão = $x 100$

(número de larvas + número de ovos) 


\section{Análise estatística}

A análise estatística foi realizada usando o software Statistix 9.0, ANOVA e comparação de médias pelo teste de Tukey $(p \leq 0,05)$.

A concentração letal média $\left(C L_{50}\right)$, concentração capaz de inibir $50 \%$ da eclosão foi determinada a partir da curva dose-resposta. Com intervalo de confiança de $95 \%$, utilizando o programa GraphPad Prism para Windows, versão 5.0.

\section{RESULTADOS E DISCUSSÃO}

As larvas infectantes foram identificadas na coprocultura como $97 \%$ de Haemonchus contortus e 3\% de Trichostrongylus spp., este resultado confirma a importância destes dois gêneros, pertencentes a família Trichostrongylidae, sendo o $H$. contortus considerado o mais patogênico aos ovinos em todo o Brasil (AMARANTE et al., 2004).

No presente estudo, o extrato hidroalcoólico nas concentrações de 40 e $20 \mathrm{mg} / \mathrm{mL}$ apresentou $100 \%$ de atividade de inibição dos ovos destes parasitos, mostrando uma queda gradual até a concentração de $1,25 \mathrm{mg} / \mathrm{mL}$ (Tabela 1). $\mathrm{O}$ valor da $\mathrm{CL}_{50}$ deste extrato foi de 4,797 mg/mL. Dias de Castro et al. (2013) em estudo com o extrato hidroalcoólico de Origanum vulgare, também da família Lamiaceae, observaram que, embora tenha demonstrado ação ovicida, esta ocorreu apenas na concentração de $80 \mathrm{mg} / \mathrm{mL}$ (96,7\%), quando testado contra ovos de nematódeos gastrintestinais de bovinos. Enquanto no presente estudo, a concentração de $10 \mathrm{mg} / \mathrm{mL}$ do extrato hidroalcoólico de $O$. basilicum já apresentou eficácia semelhante (98,6\%). Esta diferença pode estar relacionada à presença e concentrações dos diferentes compostos químicos entre as plantas, mesmo pertencendo à mesma família.

Tabela 1 - Percentual médio ( \pm desvio padrão) da inibição da eclosão do extrato hidroalcoólico de Ocimum. basilicum sobre nematódeos gastrintestinais de ovinos.

\begin{tabular}{|c|c|c|c|c|c|c|c|c|}
\hline \multirow[b]{2}{*}{ Tratamento } & \multicolumn{8}{|c|}{ Concentrações (mg/mL) } \\
\hline & $* \mathrm{AH}$ & 40 & 20 & 10 & 5 & 2,5 & 1,25 & Água \\
\hline $\begin{array}{l}\text { Atividade } \\
\text { Ovicida }\end{array}$ & $98,58 \pm 2,1^{A}$ & $100 \pm 0,0^{A}$ & $100 \pm 0,0^{A}$ & $98,56 \pm 0,77^{A}$ & $59,36 \pm 1,3^{B}$ & $14,01 \pm 1,7^{C}$ & $9,75 \pm 3,6^{C}$ & $9,9 \pm 1,1^{\mathrm{C}}$ \\
\hline
\end{tabular}


Quando na forma de óleo essencial, o 0 . basilicum apresentou percentual de inibição da eclosão de $100 \%$ nas concentrações de 32 a $0,5 \mathrm{mg} / \mathrm{mL}$, sendo que somente na concentração 0,25 mg/mL ocorreu uma pequena queda para $99 \%$ de eficiência (Tabela 2). A $\mathrm{CL}_{50}$ para inibição da eclosão foi de $0,2123 \mathrm{mg} / \mathrm{mL}$. Resultados similares foram descritos por Pessoa et al. (2002), com o óleo essencial de O. gratissimun, planta pertencente ao mesmo gênero, tendo obtido atividade de inibição da eclosão sobre ovos de nematódeos gastrintestinais de ovinos próximo a 100\%, até a concentração de 0,25 mg/mL.

Tabela 2 - Percentual médio ( \pm desvio padrão) da inibição da eclosão do óleo essencial de Ocimum basilicum sobre nematódeos gastrintestinais de ovinos.

Concentrações $(\mathrm{mg} / \mathrm{mL})$

\begin{tabular}{cc}
\hline Tratamento & $\begin{array}{c}\text { Atividade } \\
\text { ovicida }\end{array}$ \\
\hline${ }^{*} \mathrm{AH}$ & $98,58 \pm 2,1^{\mathrm{B}}$ \\
\hline 32 & $100 \pm 0,0^{\mathrm{A}}$ \\
\hline 16 & $100 \pm 0,0^{\mathrm{A}}$ \\
\hline 8 & $100 \pm 0,0^{\mathrm{A}}$ \\
\hline 4 & $100 \pm 0,0^{\mathrm{A}}$ \\
\hline 2 & $100 \pm 0,0^{\mathrm{A}}$ \\
\hline 1 & $100 \pm 0,0^{\mathrm{A}}$ \\
\hline 0,5 & $100 \pm 0,0^{\mathrm{A}}$ \\
\hline 0,25 & $99,05 \pm 0,41^{\mathrm{B}}$ \\
\hline Água & $9,9 \pm 1,1^{\mathrm{C}}$ \\
\hline
\end{tabular}

${ }^{*} \mathrm{AH}=$ tiabendazol a $0,025 \mathrm{mg} / \mathrm{mL}$. Letras maiúsculas distintas, na coluna, diferem significativamente $(p \leq 0,05)$.

Em estudos realizados com óleos essenciais de plantas de diferentes famílias, a eficácia próxima a $100 \%$ foi obtida com concentrações maiores que as do presente estudo, como detectado com os óleos essenciais de Croton zehntneri e Lippia sidoides, que demonstraram $100 \%$ de inibição da eclosão de ovos na concentração de $1,25 \mathrm{mg} / \mathrm{mL}$. (CAMURÇAVASCONCELOS et al., 2007). Esta diferença pode ser resultado da composição, atividade e concentração de compostos destas plantas com potencial anti-helmíntico.

Tendo em vista a ampla biodiversidade da flora brasileira, estudos com plantas buscando produtos naturais com atividade biológica são de fundamental importância para o desenvolvimento de novos fármacos anti-helmínticos (ASSIS et al., 2003). Assim a avaliação in vitro é amplamente utilizada por possibilitar a obtenção de resultados rápidos, com 
menores gastos, e, sobretudo evitar o uso indiscriminado de animais de experimentação (CAMURÇA-VASCONCELOS et al., 2005).

O teste de citotoxicidade realizado com o óleo essencial demonstrou que em todas as concentrações testadas foram obtidas entre $50 \%$ e $55 \%$ de células viáveis, na concentração de $0,12 \mathrm{mg} / \mathrm{mL}$ houve um aumento do número de células viáveis, atingindo $72 \%$ (Tabela 3 ).

Tabela 3 - Percentual médio de células de rim bovino Madin-Darby (MDBK) viáveis após tratamento com diferentes concentrações de óleo essencial de Ocimum basilicum.

\begin{tabular}{lcccccccccc}
\hline \multicolumn{10}{c}{ Concentração $(\mathrm{mg} / \mathrm{mL})$} \\
\hline $\begin{array}{l}\text { Tratamento } \\
\text { Percentual }\end{array}$ & Água & 32 & 16 & 8 & 4 & 2 & 1 & 0,5 & 0,25 & 0,12 \\
$\begin{array}{l}\text { de células } \\
\text { viáveis }\end{array}$ & 97,37 & 55 & 52 & 55 & 57 & 50 & 52 & 51 & 51 & 72 \\
\hline
\end{tabular}

Os resultados obtidos nos testes in vitro mostraram que ambos os extratos avaliados apresentaram eficiência na inibição da eclosão, sendo que o óleo essencial de $O$. basilicum manteve seu potencial em todas as concentrações utilizadas, já o extrato hidroalcoólico nas concentrações de $5 \mathrm{mg} / \mathrm{mL}$ ou menores, não foi efetivo. Essa diferença de eficácia pode estar relacionada aos constituintes presentes e à concentração dos mesmos nos extratos, visto que os métodos de obtenção desses extratos são diferentes. Na análise da $\mathrm{CL}_{50}$, podemos observar que o óleo essencial atingiu $50 \%$ de ação na inibição da eclosão na concentração de $0,2123 \mathrm{mg} / \mathrm{mL}$, ou seja, uma concentração 22,6 vezes menor quando comparado ao extrato hidroalcoólico.

Estudos com extratos de plantas pertencentes à família de $O$. basilicum tem mostrado ação anti-helmíntica promissora (DIAS DE CASTRO et al., 2013; PESSOA et al., 2002). Entretanto muitas são as variáveis que podem interferir na ação final de um extrato, como: época de colheita da planta, grau de umidade, parte da planta utilizada, métodos de extração, tipo de plantio, dentre outros (DAOUK et al., 1995). Segundo Powers et al. (1982), a classificação do índice de eficácia de um anti-helmíntico é dividida em: efetivo, quando ação está acima de 90\%; moderadamente efetivo, entre 80 a 90\%; pouco efetivo, entre 60 e $80 \%$; e não efetivo, abaixo de $60 \%$. Sendo assim, os resultados aqui apresentados mostram que o óleo foi efetivo em todas as concentrações testadas, enquanto que o extrato hidroalcoólico foi 
efetivo nas concentrações de $10 \mathrm{mg} / \mathrm{mL}, 20 \mathrm{mg} / \mathrm{mL}$ e $40 \mathrm{mg} / \mathrm{mL}$, pouco efetivo na concentração de $5 \mathrm{mg} / \mathrm{mL}$ e as demais concentrações não foram efetivas em inibir a eclosão.

Visto que o óleo essencial de $O$. basilicum demonstrou ação anti-helmíntica mais promissora, foi realizada análise química (Tabela 4) que identificou como constituintes majoritários o chavicol $(34,68 \%)$ e o $\beta$-linalool $(27,68 \%)$. Resultados próximos aos obtidos na análise do óleo essencial foram descritos sobre diferentes espécies de Ocimum cultivadas no Brasil, nos quais os componentes majoritários foram chavicol (36,81 a 41,62\%) e linalool (29,50 a 32,26\%) (OLIVEIRA et al., 2013).

Tabela 4 - Análise química do óleo essencial de Ocimum basilicum por cromatografia gasosa acoplada a espectrometria de massa.

\begin{tabular}{cc}
\hline Compostos & $\%$ \\
\hline 1,8 Cineol (eucalyptol) & 6,72 \\
\hline Beta linalool & 27,68 \\
\hline Chavicol (estragole) & 34,68 \\
\hline Ácido 2-propenóico, 3-fenil-, éster metílico (ácido cinâmico, éster metílico) & 16,08 \\
\hline Alfa bergamotene & 8,77 \\
\hline Gama-candineno & 6,07 \\
\hline
\end{tabular}

O chavicol está presente em diversas plantas pesquisadas, como em Feronia limonia, que apresenta o chavicol como principal constituinte (34,69\%), e quando testada contra larvas dos mosquitos Anopheles stephensi, Aedes aegypti e Culex quinquefasciatus, alcançou excelentes resultados (SENTHILKUMA et al., 2013). Quanto à ação ovicida sobre $H$. contortus, C. zehntneri contendo $21,84 \%$ de chavicol também mostrou resultados promissores (CAMURÇA-VASCONCELOS et al., 2007).

Almeida et al. (2007), testaram o linalool, segundo maior constituinte do óleo essencial de $O$. basilicum, na concentração de $300 \mu \mathrm{g} / \mathrm{mL}$, causando a morte de $100 \%$ dos trofozoítos de Giardia lamblia, enquanto que $2 \mathrm{mg} / \mathrm{mL}$ do óleo essencial mostrou ação giardicida de $80 \%$, indicando o linalool como um dos compostos com maior atividade sobre G. lamblia. No presente estudo, o linalool pode estar envolvido na ação de inibição da eclosão apresentada pelo óleo de $O$. basilicum.

Outro composto identificado nesse estudo, o 1,8 - cineol (eucaliptol), embora presente em menor concentração $(6,72 \%)$, obteve sua ação anti-helmíntica confirmada em avaliação realizada com o óleo essencial de Eucalyptus globulus, com 83,89\% deste constituinte 
(MACEDO et al., 2009). Da mesma forma, esse constituinte presente no óleo essencial de Artemisia lancea (34,6\%), mostrou ação ovicida sobre $H$. contortus, mas quando analisados separadamente, o óleo essencial e o 1,8 - cineol, apresentaram inibição da eclosão de ovos de $99,4 \%$ e $74,8 \%$, respectivamente (ZHU et al., 2013).

Embora o chavicol e o linolool sejam os principais contituintes do óleo essencial de $O$. basilicum, com o excelente resultado verificado no presente estudo, não se pode desconsiderar os demais contituintes, mesmo que em menor concentração, pois podem ocorrer ações sinérgicas entre os mesmos, com interações entre as múltiplas moléculas que agem sobre o parasito (MARIE-MAGDELEINE et al., 2009).

\section{CONCLUSÃO}

Baseado nos resultados obtidos no presente estudo pode-se concluir que, tanto o extrato hidroalcoólico como o óleo essencial de 0 . basilicum possuem atividade na inibição da eclosão sobre Haemonchus spp. e Trichostrongylus spp. de ovinos, sendo o óleo essencial mais promissor, mostrando-se eficaz em menores concentrações. Futuros estudos são necessários para avaliar a ação anti-helmíntica in vitro e in vivo dos componentes majoritários do óleo, visando uma alternativa sustentável de controle desses nematódeos.

\section{OVICIDAL ACTIVITY OF THE ESSENCIAL OIL AND THE HIDROALCOHOLIC EXTRACT OF Ocimum basilicum ON GASTROINTESTINAL NEMATODES OF SHEEP}

\section{ABSTRACT}

The control of gastrointestinal helminths in sheep, carried out exclusively through anthelmintics, results in the selection of helminth populations resistant to practically all available drugs. This has motivated the search for control alternatives, among which is highlighted the use of herbal medicines. In this context the aim of this study was to evaluate the ovicidal activity in vitro of the essential oil and the hydroalcoholic extract of Ocimum basilicum in nematodes of sheep and determine the major chemical constituents of the essential oil. For the in vitro studies feces from naturally infected sheep were tested against the $O$. basilicum essential oil in concentrations from 32 to $0.25 \mathrm{mg} / \mathrm{mL}$ and the hydroalcoholic extract at concentrations from 40 to $1.25 \mathrm{mg} / \mathrm{mL}$. Thiabendazole was used as positive control $(0.025 \mathrm{mg} / \mathrm{mL})$ and distilled water as negative control. The chemical analysis of the essential oil determined as major constituents chavicol (34.68\%) and linalool 
(27.68\%). The essential oil showed ovicidal activity above $99 \%$ at all concentrations tested. The hydroalcoholic extract at concentrations between 40 and $20 \mathrm{mg} / \mathrm{mL}$ had $100 \%$ of action, decreasing from $10 \mathrm{mg} / \mathrm{mL}$, reaching $9.76 \%$ of activity in the concentration $1.25 \mathrm{mg} / \mathrm{mL}$. Positive and negative controls obtained $98.57 \%$ and $8.5 \%$, respectively. The $\mathrm{LC}_{50}$ values of hydroalcoholic extract and essential oil were $4.797 \mathrm{mg} / \mathrm{mL}$ and $0.2123 \mathrm{mg} / \mathrm{mL}$, respectively. $O$. basilicum presents anthelmintic action in vitro on gastrointestinal nematodes of sheep, with the greatest potential for the essential oil, being a promising herbal substance for studies searching for bioactive molecules to be used in the control of gastrointestinal nematodes.

Keywords: Basil. Haemonchus. In vitro. Anthelminthic activity.

\section{ACTIVIDAD OVICIDA DEL ACEITE ESENCIAL Y EXTRACTO HIDROALCOHÓLICO DE Ocimum basilicum SOBRE NEMATODOS GASTROINTESTINALES DE OVINOS}

\section{RESUMEN}

E I control de los helmintos gastrointestinales en ovinos, realizado exclusivamente a través de antihelmínticos, tiene como consecuencia la selección de poblaciones de helmintos resistentes a prácticamente todos los fármacos disponibles. Esto ha motivado a la búsqueda de alternativas para el control, entre las que cabe destacar la utilización de fitoterapeúticos. En este contexto el objetivo de este estudio fue evaluar la actividad ovicida in vitro del aceite esencial y del extracto hidroalcohólico de Ocimum basilicum en nematodos de ovinos e identificar los componentes mayoritarios del aceite esencial. Para los estudios in vitro fueron utilizadas las heces de ovinos naturalmente infectados, probados frente al aceite esencial de $O$. basilicum en las concentraciones de 32 a $0,25 \mathrm{mg} / \mathrm{mL}$ y al extracto hidroalcohólico en las concentraciones de 40 a $1,25 \mathrm{mg} / \mathrm{mL}$, como control positivo se usó tiabendazol $(0,025 \mathrm{mg} / \mathrm{mL})$ y como control negativo agua destilada. El análisis químico del aceite esencial determinó como componentes mayoritarios el chavicol $(34,68 \%)$ y el linalool $(27,68 \%)$. El aceite esencial presentó actividad ovicida arriba del $99 \%$ en todas las concentraciones probadas. El extracto hidroalcohólico en las concentraciones de entre 40 e $20 \mathrm{mg} / \mathrm{mL}$ obtuvo 100\% de acción, disminuyendo a partir de la concentración de $10 \mathrm{mg} / \mathrm{mL}$, con $9,76 \%$ de actividad en la concentración de $1,25 \mathrm{mg} / \mathrm{mL}$. Los controles positivos y negativos obtuvieron $98,57 \%$ y $8,5 \%$, respectivamente. El valor de la $\mathrm{CL}_{50}$ del extracto hidroalcohólico y del aceite esencial fueron de $4,797 \mathrm{mg} / \mathrm{mL}$ y $0,2123 \mathrm{mg} / \mathrm{mL}$, respectivamente. El $O$. basilicum presentó acción antihelmíntica in vitro sobre huevos de nematodos gastrointestinales de ovinos, con mayor potencial el aceite esencial, siendo un promisor fitoterapeútico para estudios en la búsqueda de moléculas bioactivas para ser utilizadas en el control de los nematodos gastrointestinales.

Palabras clave: Albaca. Haemonchus. In vitro. Actividad anti helmíntica. 


\section{REFERÊNCIAS}

ALMEIDA, I.; ALVIANO, D. S.; VIEIRA, D. P.; et al. Antigiardial activity of Ocimum basilicum essential oil. Parasitology Research, v. 101, p. 443-452, 2007.

ALMEIDA, F. A.; GARCIA, K. C. O. D.; TORGERSON, P. R.; et al. Multiple resistance to anthelmintics by Haemonchus contortus and Trichostrongylus colubriformis in sheep in Brazil. Parasitology International, v. 59, p. 622-625, 2010.

AMARANTE, A. F. T.; BRICARELLO, P. A.; ROCHA, R. A.; et al. Resistance of Santa Ines, Suffolk and lle de France lambs to naturally acquired gastrointestinal nematode infections.

Veterinary Parasitology, v. 120, p. 91-106, 2004.

ASSIS, L. M.; BEVILAQUA, C. M. L.; MORAIS, S. M.; et al. Ovicidal and larvicidal activity in vitro of Spigelia anthelmia Linn extracts on Haemonchus contortus. Veterinary Parasitology, $v$. 117, n. 1-2, p. 43-49, 2003.

BOWN, D. Encyclopedia of Herbs \& Theirs Uses. New York; Dorling Kindersley Publishing, Inc., 1995. 424p.

CAMURÇA-VASCONCELOS, A. L. F.; BEVILAQUA, C. M. L.; MORAIS, S. M.; et al. Anthelmintic activity of Croton zehntneri and Lippia sidoides essential oils. Veterinary Parasitology, v. 148, n. 3-4, p. 288-294, 2007.

CAMURÇA-VASCONCELOS, A. L. F.; MORAIS, S. M.; SANTOS, L. F. L. Validação de plantas medicinais com atividade anti-helmíntica. Revista Brasileira de Plantas Medicinais, v. 7, n. 3, p. 97-106, 2005.

CARVALHO, C. O.; CHAGAS, A. C. S.; COTINGUIBA, F.; et al. The anthelmintic effect of plant extracts on Haemonchus contortus and Strongyloides venezuelensis. Veterinary

Parasitology, v. 183, p. 260-268, 2012.

DAMBOLENA, J. S.; ZUNINO, M. P.; LOPEZ, A. G.; et al. Essential oils composition of Ocimum basilicum L. and Ocimum gratissimum L. from Kenya and their inhibitory effects on growth and fumonisim production by Fusarium verticillioides. Innovative Food Science and Emmerging Technologies, v. 11, p. 410-414, 2010.

DAOUK, R. K.; DAGHER, S. M.; SATTOUT, E. J. Antifungal Activity of the Essential Oil of Origanum syriacum L. Journal of Food Protection, v. 58, n. 10, p. 1147-1149, 1995.

DIAS DE CASTRO, L. L.; MADRID, I. M.; AGUIAR, C. L. G.; et al. Origanum vulgare (Lamiaceae) ovicidal potential on gastrointestinal nematodes of cattle. Ciência Animal Brasileira, v. 14, n. 4, p. 508-513, 2013. 
ECHEVARRIA, F.; BORBA, M. F. S.; PINHEIRO, A. C.; et al. The prevalence of anthelmintic resistance in nematode parasites of sheep in Southern Latin America: Brazil. Veterinary Parasitology, v. 62, p. 199-206, 1996.

GHOSH, A.; CHOWDHURY, N.; CHANDRA, G. Plant extracts as potential mosquito larvicides. Indian Journal of Medical Research, v. 135, p. 581-598, 2012.

GITHIGIA, S. M.; THAMSBORG, S. M.; MUNYUA, W. K. Impact of gastrintestinal helminths on production in goats in Kenia. Small Ruminant Research, v. 42, p. 21-29, 2001.

GITHIORI, J. B.; HÖGLUND, J.; WALLER, P. J.; BAKER, R. L. Anthelmintic activity of preparations derived from Myrsine africana and Rapanea melanophloeos against the nematode parasite, Haemonchus contortus, of sheep. Journal of Ethnopharmacology, v. 80, n. 2-3, p. 187-191, 2002.

GORDON, H. M.; WHITLOCK, H. V. A new technique for counting nematode eggs in sheep faeces. Journal of the Council for Scientific and Industrial Research, v. 12, p. 50-52, 1939.

HUBERT, J.; KERBOEUF, D. A. Microlarval development assay for the detection of anthelmintic resistance in sheep nematodes. Veterinary Research, v. 130, n. 20, p. 442-446, 1992.

MACEDO, I. T. F.; BEVILAQUA, C. M. L.; OLIVEIRA, L. M. B.; et al. Atividade ovicida e larvicida in vitro do óleo essencial de Eucalyptus globulus sobre Haemonchus contortus. Revista Brasileira de Parasitologia Veterinária, v. 18, n. 3, p. 62-66, 2009.

MARIE-MAGDELEINE, C.; HOSTE, H.; MAHIEU, M.; et al. In vitro effects of Cucurbita moschata seed extracts on Haemonchus contortus. Veterinary Parasitology, v. 161, p. 99105, 2009.

MOSMANN, T. Rapid colorimetrie assay for cellular growth and survival: application to proliferation and cytotoxicity assays. Journal of Immunological Methods, v. 65, p. 55-63, 1983.

MOTA, M. L.; LOBO, L. T.; COSTA, J. M.; et al. In Vitro and In Vivo Antimalarial Activity of Essential Oils and Chemical Components from Three Medicinal Plants Found in Northeastern Brazil. Planta Medica, v. 78, n. 7, p. 658-664, 2012.

OLIVEIRA, L. M. B.; BEVILAQUA, C. M.; COSTA, C. T. Anthelmintic activity of Cocos nucifera L. against sheep gastrointestinal nematodes. Veterinary Parasitology, v. 159, p. 55-59, 2009.

OLIVEIRA, R. A.; MOREIRA, I. S.; OLIVEIRA, F. F. Linalool and methyl chavicol present basil (Ocimum sp.) cultivated in Brazil. Revista Brasileira de Plantas Medicinais, v. 15, n. 2, p. 309-311, 2013. 
PESSOA, L. M.; MORAIS, S. M.; BEVILAQUA, C. M. L. Anthelmintic activity of essential oil of Ocimum gratissimum Linn. and eugenol against Haemonchus contortus. Veterinary

Parasitology, v. 109, n. 1-2, p. 59-63, 2002.

POWERS, K. G.; WOOD, L. B.; ECKERT, J.; et al. World Association for the Advancement of Veterinary Parasitology (W. A. A. V. P.) guidelines for evaluating the efficacy of anthelmintics in ruminantes (bovine and ovine). Veterinary Parasitology, v. 10, n. 4, p. 265-284, 1982.

ROBERTS, F. H. S.; O'SULLIVAN, P. J. Methods for egg counts and larvae cultures for strongyles infesting the gastrointestinal tract of cattle. Australian Journal of Agricultural Research, v. 1, p. 99-102, 1950.

SENTHILKUMA, A.; JAYARAMAN, M.; VENKATESALU, V. Chemical constituents and larvicidal potential of Feronia limonia leaf essential oil against Anopheles stephensi, Aedes aegypti and Culex quinquefasciatus. Parasitology Research. v. 112, p. 1337-1342, 2013.

ZHU, L.; DAÍ, J. L.; YANG, L.; QIU, J. In vitro ovicidal and larvicidal activity of the essential oil of Artemisia lancea against Haemonchus contortus (Strongylidae). Veterinary Parasitology, v. 195, p. 112-117, 2013.

Autor para correspondência: Leonardo Mortagua de Castro. Universidade Federal de Pelotas, Faculdade de Veterinária, Campus Universitário, Capão do Leão (RS), Brasil. leonardomortagua@gmail.com 